KOMPASS

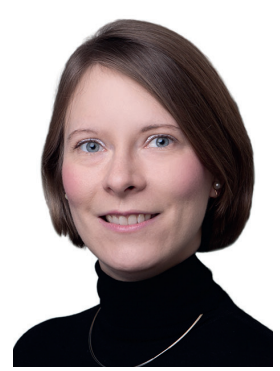

Nina Neuendorff

Koordination «OnkoCampus»

\title{
Engagement für Weiterbildung, Forschung und darüber hinaus
}

Liebe Leserinnen und Leser, herzlich willkommen! Wir hoffen, Ihnen auch in der aktuellen Ausgabe wieder interessante Themen präsentieren zu dürfen.

Viele junge Ärztinnen und Ärzte werden Mitglied bei einer Fachgesellschaft, da es meist mit vielen Vorzügen wie beispielsweise ermäßigten Teilnahmegebühren bei Fortbildungen und Jahrestagungen verbunden ist. Doch darüber hinaus gibt es in den meisten Fachgesellschaften auch Nachwuchsgruppen, in denen man sich engagieren kann. Was dies bedeutet und mit welchen Chancen dies verbunden ist, berichten uns die Vertreterinnen und Vertreter einiger hauptsächlich onkologischer Fachgesellschaften. Anhand des Beispiels der Arbeitsgruppe junge DEGRO fassen wir außerdem zusammen, welche Aufgaben eine Nachwuchsgruppe innerhalb einer Fachgesellschaft wahrnehmen kann und warum das für die Zukunft eines Fachgebiets so wichtig ist.

Weiterhin stellen wir in einem kurzen Interview mit Fabian Buslaff die MedizinstudentenInitiative «Aufklärung gegen Tabak e.V.» vor, welche sich seit 2012 aktiv für die Verbreitung von Methoden der Tabakentwöhnung engagiert. Die Initiative zieht schon weite Kreise: Heute klären über 1500 Medizinstudierende von 28 deutschen, 4 österreichischen und 2 Schweizer Universitäten pro Jahr ehrenamtlich 23800 Schüler über die Folgen des Rauchens auf.

Wohl kaum jemand beschäftigt sich gerne mit den juristischen Fallstricken des ärztlichen Alltags. Der Jurist Maximilian Broglie informiert uns in einem Beitrag über das Übernahmeverschulden.

Nun wünsche ich Ihnen viel Spaß beim Lesen und scheuen Sie sich nicht, uns bei Anregungen oder Kritik zu kontaktieren, vielleicht gibt es ja auch Themenwünsche?

\section{KARGER}

(C) 2019 S. Karger GmbH, Freiburg 


\section{Berufsverbände \\ Junge Ärztinnen und Ärzte engagieren sich für Beruf und Netzwerk}

Deutschland wird alt. Das trifft nicht nur auf die Bevölkerung Deutschlands allgemein zu, auch der Altersdurchschnitt der Ärztinnen und Ärzte in Deutschland steigt. 2017 waren laut statistischem Bundesamt 68\% der in Praxen tätigen Ärztinnen und Ärzte mindestens 50 Jahre alt. Der Anteil aller berufstätigen Ärztinnen und Ärzte in Deutschland unter 35 lag im selben Jahr bei 18,9\%. Der gehobene Altersdurchschnitt macht sich natürlich auch innerhalb der Fachgesellschaften bemerkbar. Die Themen, mit denen sich die verschiedenen Altersgruppen beschäftigen, gehen immer weiter auseinander. Engagierte junge Ärztinnen und Ärzte organisieren sich daher verstärkt in eigenen Netzwerken. Bei uns stellen 4 Vertreter «der Jungen» ihre Organisation vor und erklären, warum es sich lohnt, dabei zu sein.

\section{Die Junge DGHO}

Die Junge DGHO wurde im Oktober 2018 als Arbeitskreis der Deutschen Gesellschaft für Hämatologie und Medizinische Onkologie (DGHO) gegründet. Sie hat sich zum Ziel gesetzt, die Interessen junger, in der Hämatologie und Onkologie tätiger Kolleginnen und Kollegen innerhalb der Fachgesellschaft und nach außen zu vertreten. Die Junge DGHO soll ein Netzwerk zur Förderung des aktiven Austausches und eine Plattform zur Erfassung, Formulierung und Vertretung gesundheitspolitischer und berufsgruppenspezifischer Interessen junger Kolleginnen und Kollegen sein und zur aktiven Mitgestaltung motivieren.

Aktuell sind insbesondere die neue (Muster-)Weiterbildungsordnung (MWBO), die Anerkennung von Forschungszeiten in der Facharztausbildung und damit übergreifend die Vereinbarkeit von Klinik und Forschung sowie grundlegend die Vereinbarkeit von Privatleben und Beruf von großem Interesse.

Um sich Gehör zu verschaffen, gestaltet die Junge DGHO eigene Veranstaltungen auf den Jahrestagungen der Deutschen, Österreichischen und Schweizerischen Gesellschaft für Hämatologie und Medizinische Onkologie, publiziert Beiträge in den Mitgliederrundschreiben, wird aktiv in die Juniorakademie und den Studententag der DGHO eingebunden und in ihren Aktivitäten wirksam durch die DGHO Service GmbH unterstützt. Damit bestehen bereits beste Voraussetzungen für eine erfolgreiche Arbeit des Arbeitskreises, und wir können die weiteren Entwicklungen der Jungen DGHO mit Spannung erwarten.

Ein Engagement in Fachgesellschaften wie der DGHO ist in vielerlei Hinsicht vorteilhaft. Allem voran bietet sich so die Möglichkeit, persönlich aktiv zu werden und gemeinsam mit anderen motivierten Kolleginnen und Kollegen die Arbeit der Fachgesellschaften hautnah zu erleben, zu gestalten und im Hinblick auf die eigenen Belange zu verbessern. Aus diesem Grunde haben wir uns gemeinsam entschlossen, den Arbeitskreis Junge DGHO ins Leben zu rufen und sind mit unserem Impuls wohlwollend und unterstützend in der DGHO empfangen worden.

Durch die Gründung des Arbeitskreises Junge DGHO haben wir die Möglichkeit bekommen, uns mit den Führungskräften und Ent-

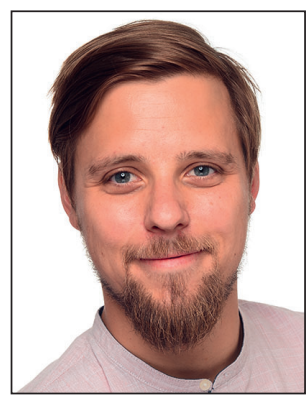

Dr. Christoph Oing

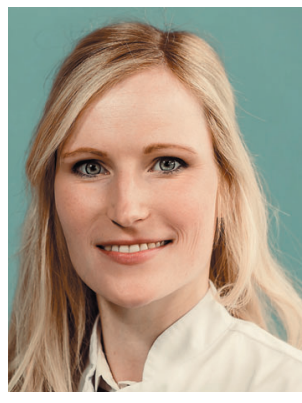

Dr. Caroline Anna Peuker

scheidungsträgerinnen und -trägern unseres Fachgebietes bekannt zu machen, dadurch wertvolle Kontakte zu knüpfen, bereits bestehende Netzwerke zu nutzen und neue, für uns wichtige Netzwerke zu schaffen.

Die Junge DGHO bietet als Plattform und Interessengemeinschaft eine großartige Gelegenheit für junge Kolleginnen und Kollegen die Aktivitäten und Ziele unserer Fachgesellschaft, die unlängst im Positionspapier der DGHO formuliert worden sind, zu unterstützen und zukünftig mitzugestalten. Nur wer selbst aktiv wird, kann etwas bewegen!

Dr. Caroline Anna Peuker und Dr. Christoph Oing, Sprecher des Arbeitskreises Junge DGHO (https://www.dgho.de/arbeitskreise/i-k/junge_dgho)

\section{Das Bündnis Junge Ärzte (BJÄ)}

Das Bündnis Junge Ärzte setzt sich aus den Assistentensprecherinnen und -sprechern der größten deutschen Berufs- und Fachverbände zusammen. Unsere Grundidee ist, gemeinsame Probleme gemeinsam anzusprechen. Um das zu tun, sind wir an vielen Stellen aktiv - von den Kammern bis zur Politik. So haben wir uns vor einigen Jahren z.B. erfolgreich dafür stark gemacht, dass es ein Forum für junge Ärztinnen und Ärzte auf dem Deutschen Ärztetag gibt. Die Situation in den Krankenhäusern wird weder den Patienten noch den Ärztinnen und Ärzten gerecht. Wenn wir wollen, dass sich die Rahmenbedingungen mehr in die Richtung entwickeln, die uns vorschwebt, müssen wir uns auch dafür einsetzen. Ich habe durch meinen Einsatz im BJÄ persönlich viel gelernt und habe viele spannende Dinge erlebt. Ob es meine Karriere vorangebracht hat - fraglich. Ob es mich persönlich vorangebracht hat - definitiv!

Dr. Kevin Schulte, Sprecher des Bündnis Junge Ärzte (https://www.buendnisjungeaerzte.org/home/)

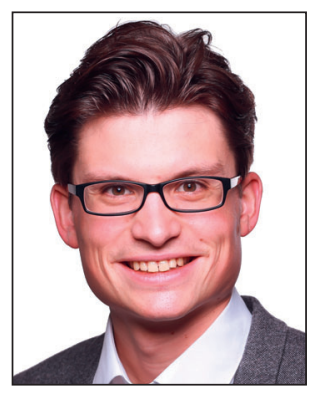

Dr. Kevin Schulte 


\section{Die Young SIOG Interest Group}

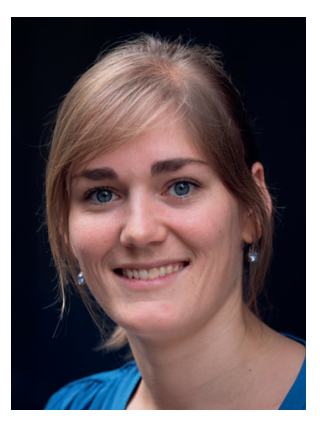

Nienke De Glas

Die Young SIOG Interest Group hat sich zum Ziel gesetzt, ein internationales Netzwerk aus jungen Ärzten und Wissenschaftlern im Bereich der geriatrischen Onkologie aufzubauen, um so internationale Kooperationen zu stärken. Die Gruppe organisiert einige Programmpunkte während der jährlichen Konferenz der International Society of Geriatric Oncology (SIOG) und setzt sich für frühe Integration der Mitglieder innerhalb der SIOG ein. Alle 3 Monate findet eine Young-SIOG-Telefonkonferenz statt, in der laufende oder geplante Forschungsprojekte der Mitglieder oder klinische Fälle diskutiert werden. Zusätzlich publizieren wir regelmäßig auf dem Feld der geriatrischen Onkologie.

Ich habe diese Gruppe 2013 mit einer Kollegin gegründet. In den vergangenen Jahren sind wir zu einer großen Gruppe aus hochmotivierten jüngeren Leuten zusammengewachsen. Für mich war die Gründung der Gruppe eine prägende Erfahrung. Es war überwältigend zu sehen, wie die Gruppe zu ihrer heutigen Form gewachsen ist. Außerdem hat es mir die Möglichkeit verschafft, mit renommierten Ärzten und Wissenschaftlern auf diesem Feld in engen
Kontakt zu kommen. Ich kann ein Engagement in unserer Gruppe nur allen interessierten jüngeren Wissenschaftlerinnen und Wissenschaftlern sowie Ärztinnen und Ärzten mit Interesse an der geriatrischen Onkologie empfehlen, denn man wächst in das bestehende Netzwerk hinein, erwirbt Führungserfahrung und kann intensiv voneinander lernen.

Nienke De Glas, Vorsitzende der Young SIOG Interest Group (http://siog.org/content/young-siog-interest-group)

\section{Das Young Oncologists Committee der ESMO}

Das Young Oncologists Committee (YOC) der European Society for Medical Oncology (ESMO) fokussiert sich auf Thematiken von Nachwuchsmedizinerinnen und -medizinern innerhalb der ESMO und stellt sicher, dass die Aktivitäten und der Fokus der Gesellschaft diese berücksichtigen. Ein wichtiger Fokus ist die Kongressorganisation. Das YOC der ESMO organisiert in jedem Jahr eigene Inhalte speziell für Nachwuchsmedizinerinnen und -mediziner. Hier werden wichtige Thematiken für den klinischen Alltag, aber auch Mentoring und Karriereentwicklung adressiert. Zudem gibt es eine eigene Session für Medizinstudierende, um auch bereits diese in die Gesellschaft zu integrieren und für sie relevante Inhalte anzubieten.

Das YOC der ESMO ist eine wunderbare Möglichkeit mit Kollegen aus ganz Europa zusammenzuarbeiten. So haben sich für mich neben der spannenden Arbeit für die ESMO neue Freundschaften entwickelt. Vor allem sind daraus auch Forschungsprojekte hervorgegangen, an denen wir institutsübergreifend gemeinsam arbeiten. Ich kann daher jedem nur empfehlen, sich im Young Oncologists Committee der ESMO zu engagieren.

Dr. Anna Berghoff, Ausschussmitglied der YOC der ESMO (https://www. esmo.org/About-Us/Who-We-Are/Young-Oncologists-Committee)

\section{Weiterbildung und Forschung \\ Die Zukunft der Radioonkologie aktiv gestalten - die AG junge DEGRO}

In den letzten Jahrzehnten hat sich die medizinische (Arbeits-)Welt und mit ihr die Einstellungen und Erwartungen junger Ärzte signifikant verändert [1, 2]. Umso wichtiger ist es, die Anliegen der jungen Generation konzertiert und effektiv zu artikulieren.

Innerhalb der Deutschen Gesellschaft für Radioonkologie (DEGRO) nimmt die AG junge DEGRO (jDEGRO) diese Aufgabe erfolgreich wahr. Als Zusammenschluss junger Strahlentherapeut/inn/en, Medizinphysiker/innen und Strahlenbiolog/inn/en versteht sie sich als offenes Netzwerk, das den forschungsinteressierten Nachwuchs aus den eigenen Reihen fördern sowie Weiterbildung und Forschung optimieren will.

Im Hinblick auf klinische Forschungsprojekte gelang der AG die erfolgreiche Bildung eines deutschlandweiten Forschungsnetzwerks, das in einem ersten Projekt die Bestrahlung des inoperab- len nicht kleinzelligen Lungenkarzinoms (NSCLC) im Stadium III untersuchte: Hierbei interessierte die Studiengruppe die prognostische Aussagekraft des Tumorvolumens (gross tumor volume, GTV), das bei der Radiotherapieplanung auf Grundlage der CT- bzw. PET-CT-Bildgebung definiert wird. Vorab wurden in einer systematischen Literaturrecherche Vorarbeiten identifiziert und analysiert [3].

An der folgenden multizentrischen Studie (NCT03055715; ARO2017-01) beteiligten sich 21 Zentren, eingeschlossen waren darin 346 Patienten [4]. Mittels standardisierter elektronischer «case report forms» wurden das initiale GTV sowie seine Änderung im Verlauf erfasst. Dies erfolgte anhand einer weiteren Schnittbildgebung vor Beginn der Boost-Bestrahlung (nach etwa 5 Wochen Therapie), anhand derer ein zweites GTV eingezeichnet wurde. 


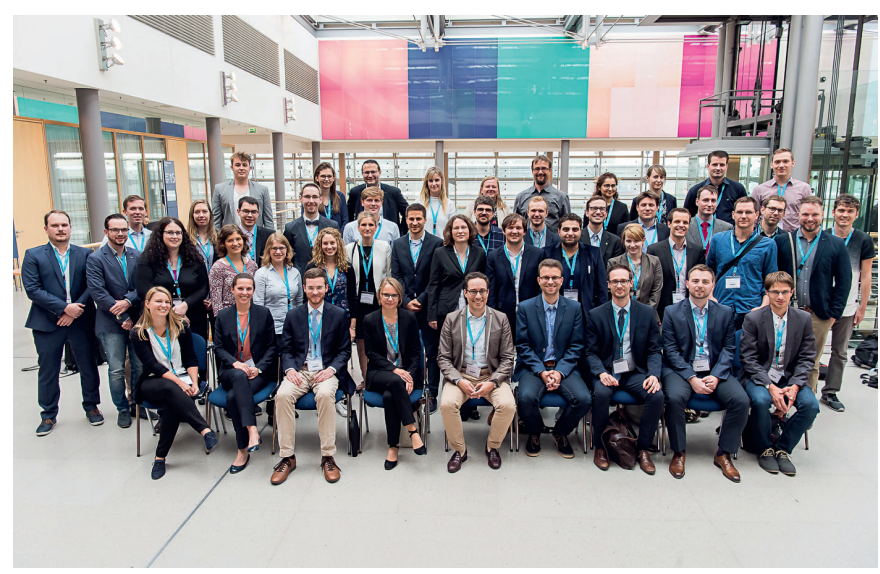

Gruppenfoto der jDEGRO auf der Jahrestagung der Deutschen Gesellschaft für Radioonkologie (Leipzig 2018)

Neben dem GTV zu Beginn der Bestrahlung hatte v.a. das im Verlauf der Radiotherapie neu erstellte GTV einen signifikanten Einfluss auf das Gesamtüberleben, unabhängig von anderen Prognosefaktoren wie dem T- oder N-Stadium. Auch die mittels Mediansplit eingeteilte absolute Volumenreduktion vom ersten zum zweiten GTV hatte einen signifikanten Einfluss auf das Gesamtüberleben [5].

Dies zeigt die prognostische Wertigkeit des (intratherapeutischen) GTV bei inoperablen NSCLC-Patienten. Ausgehend hiervon sind aufbauende Analysen unter Einschluss der Bilddaten und Bestrahlungspläne geplant.

Neben der (klinischen) Forschung gehört es zu den AG-Aufgaben, die Jahrestagungen der DEGRO mitzugestalten: Mitglieder der jDEGRO sind stets Teil des Organisationsteams und vermitteln die Beteiligung am wissenschaftlichen Programm. Zusammen mit den Kongresspräsidenten werden die Themen ausgewählt, das Programm konzipiert und organisatorische Details diskutiert. Zusätzlich werden durch Mitglieder der jDEGRO Tagungsbeiträge begutachtet, wissenschaftliche Sitzungen moderiert und eigene Veranstaltungen organisiert. DiejDEGRO deckt damitvielfältige Aufgaben $a b$, die wichtig sind, um den Jahreskongress für «alte Hasen» und «Neulinge» der Strahlentherapie in gleichem Maße interessant zu gestalten

Weiterhin beteiligt sich die jDEGRO daran, die Facharztausbildung und das Fortbildungsangebot stetig weiterzuentwickeln. Eine deutschlandweite Umfrage unserer AG zur Facharztausbildung in der Strahlentherapie zeigte, dass junge Kolleg/inn/en insgesamt zufrieden in ihrem Fach sind und die Qualität der Weiterbildung schätzen [6]. Insbesondere bei Spezialtechniken und seltenen Indikationen wünschten sich jedoch viele Kolleg/inn/en weitere Angebote, sodass ein deutschlandweites Hospitationsprogramm zum Zwecke der fokussierten Weiterbildung in diesen Bereichen in Planung ist. Zusätzlich läuft aktuell eine Umfrage zum Stand der strahlentherapeutischen Lehre an den deutschen Universitätskliniken, auch in Hinblick auf die Entwicklung des Nationalen Lernzielkatalogs Medizin und die Umsetzung des Masterplans Medizinstudium 2020.

Diese vielfältigen Aufgaben werden in den 3 jährlichen AG-Sitzungen der jDEGRO und regelmäßigen Telefonkonferenzen bespro-

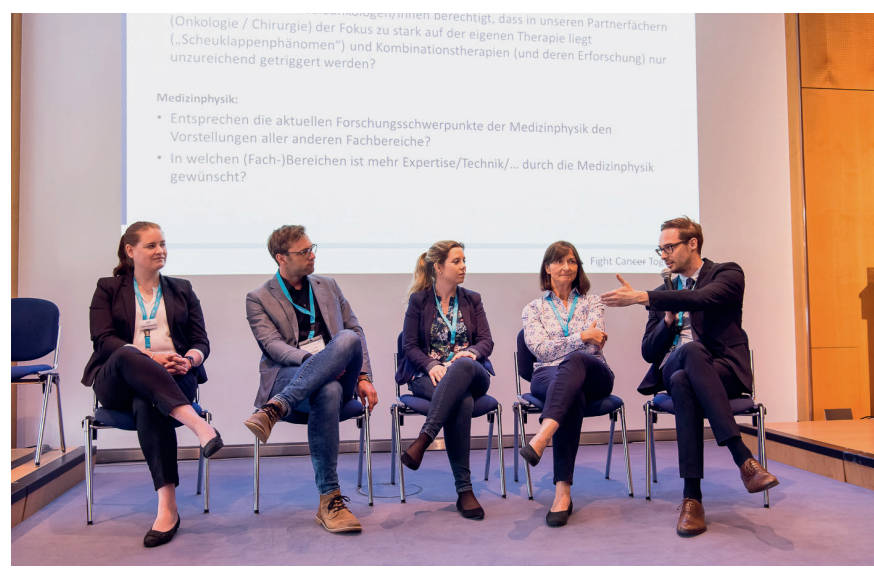

Im Symposium der AG jDEGRO «Young Professionals Fight Cancer - Perspektiven von Nachwuchsgruppen in der Onkologie» auf dem Jahreskongress 2018 trafen sich Vertreter der aufstrebenden Nachwuchsgruppen aus der Medizin, Biologie und Physik um die jeweiligen Perspektiven und Beiträge zur Krebstherapie darzustellen und eine lebendige Diskussion zur interdisziplinären Onkologie zu führen.

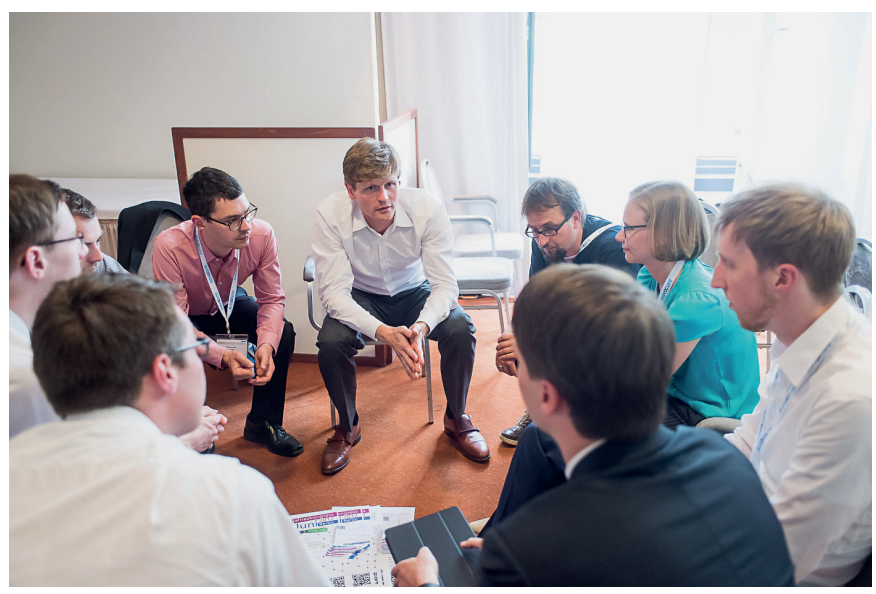

Teamwork im Rahmen der AG-Sitzung der jDEGRO

chen und koordiniert. In den angeregten Diskussionen werden konstruktive Lösungen im kollegialen Austausch erarbeitet. Das bisher Erreichte ist für die Gruppe Ansporn, an der Zukunft des Faches Strahlentherapie weiterhin engagiert gestaltend mitzuarbeiten.

\section{Zusammenfassung}

Der AG jDEGRO gelang mit der multizentrischen Studie zur GröBenänderung des Tumorvolumens von NSCLC-Patienten unter Radiotherapie die Etablierung eines leistungsstarken Forschungsnetzwerkes junger Strahlentherapeut/inn/en, -biolog/ inn/en und -physiker/innen. Primäre Aufgabe und Anspruch der AG ist es, Forschung und Weiterbildung in der Radioonkologie innerhalb der Fachgesellschaft aktiv mitzugestalten und die Interessen der Nachwuchswissenschaftler/innen zu vertreten und zu fördern.

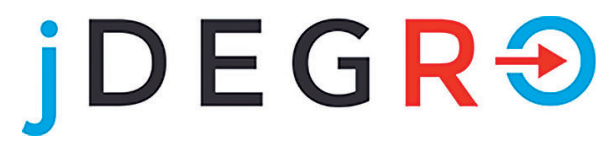




\section{Literatur}

1 Boysen PG, Daste L, Northern T: Multigenerational Challenges and the Future of Graduate Medical Education. Ochsner J 2016;16:101-107.

2 Desy JR, Reed DA, Wolanskyj AP: Milestones and Millennials: A Perfect PairingCompetency-Based Medical Education and the Learning Preferences of Generation Y. Mayo Clin Proc 2017;92:243-250.

3 Käsmann L, Niyazi M, Blanck O, et al.: Predictive and prognostic value of tumo volume and its changes during radical radiotherapy of stage III non-small cell lung cancer : A systematic review. Strahlenther Onkol 2018;194:79-90.

4 Ostheimer C, Baues C, Baumann R, et al.: Predictive value of GTV in radiotherapy of NSCLC - early results of the NCT03055715 trial. Radiotherapy and Oncology 2018;127,S175,4OC-0329.

5 Ostheimer C, jDEGRO Trial Group: Prognostischer Wert des Tumorvolumens in der Radiochemotherapie des lokal fortgeschrittenen nichtkleinzelligen Bronchialkarzinoms. Pilotstudie der AG Junge DEGRO der Deutschen Gesellschaft für Radioonkologie (DEGRO). FORUM 2018;33:446-447.
6 Dietzel CT, Jablonska K., Niyazi M, et al.: Quality of training in radiation oncology in Germany: where do we stand?: Results from a 2016/2017 survey performed by the working group «young DEGRO» of the German society of radiation oncology (DEGRO). Strahlenther Onkol 2018;194:293-302.

Kontakt

Dr. Michael Oertel, Klinik für Strahlentherapie - Radioonkologie, Universitätsklinikum Münster, Albert-Schweitzer-Campus 1, Gebäude A1, 48149 Münster, michael.oertel@ukmuenster.de.

Dr. Daniel F. Fleischmann, Klinik und Poliklinik für Strahlentherapie und Radioonkologie, Klinikum der Universität München, LMU München.

Dr. Christian Ostheimer: Klinik und Poliklinik für Strahlentherapie, MartinLuther-Universität Halle-Wittenberg, Halle (Saale).

Dr. Matthias Mäurer: Klinik für Strahlentherapie und Radioonkologie, Universitätsklinikum Jena, Friedrich-Schiller-Universität Jena.

\section{Medizinstudenten engagieren sich}

\section{«Aufklärung gegen Tabak e.V.»}

Im Verein «Aufklärung gegen Tabak e.V.» bieten Medizinstudenten an vielen Fakultäten ein Wahlfach an, in dem angehende Ärztelernen, ihre Patienten bei der Rauchentwöhnung zu unterstützen. Was der Verein noch alles macht, erklärt Vorstandsmitglied Fabian Buslaff im Interview mit Operation Karriere.

Ein höheres Risiko für Lungenkrebs und Herz-Kreislauf-Erkrankungen, aber auch ein schwächeres Immunsystem und eine schlechte Haut: Wer raucht, schadet damit seiner Gesundheit. Das ist grundsätzlich bekannt - trotzdem raucht immer noch etwa jeder vierte Deutsche. Fabian Buslaff ist im Verein «Aufklärung gegen Tabak e.V.» aktiv, um Methoden der Rauchentwöhnung zu verbreiten.

\section{Herr Buslaff, warum ist es denn so wichtig, über die Folgen des} Rauchens aufzuklären?

Das Rauchen ist in Deutschland immer noch sehr verbreitet. Auch wenn man heute in den Medien liest, dass die Jugend immer weniger raucht, ist es tatsächlich noch so, dass etwa jede fünfte Frau und jeder dritte Mann regelmäßig zur Zigarette greift, also etwa ein Viertel der Deutschen. Das spürt man natürlich gerade in gesundheitsnahen Berufen ganz besonders: Wenn wir als Medizinstudenten im Krankenhaus sind, sehen wir häufig Patienten, die an den Folgen des Rauchens leiden.

\section{Um was für Folgeerkrankungen geht es da genau?}

Das, was den meisten zuerst in den Sinn kommt, sind natürlich Folgeerkrankungen, die direkt die Atemwege betreffen. Das sind zum Beispiel die Chronisch-obstruktive Lungenerkrankung COPD, die quasi jeder irgendwann bekommt, der über viele Jahre geraucht hat. Lungenkrebs ist natürlich sehr bekannt - dabei ist er gar nicht der häufigste Faktor, der bei Rauchern zum Tod führt. Vielen ist nicht bewusst, dass der Effekt auf das Herz-Kreislauf-System für viel mehr Todesfälle und Erkrankungen von Rauchern verantwortlich ist. Da geht es zum Beispiel um Erkrankungen wie Arteriosklerose, die letztendlich sehr häufig zum Herzinfarkt führen. Nicht jeder kennt jemanden, der Lungenkrebs bekommen hat. Aber Schlagan-

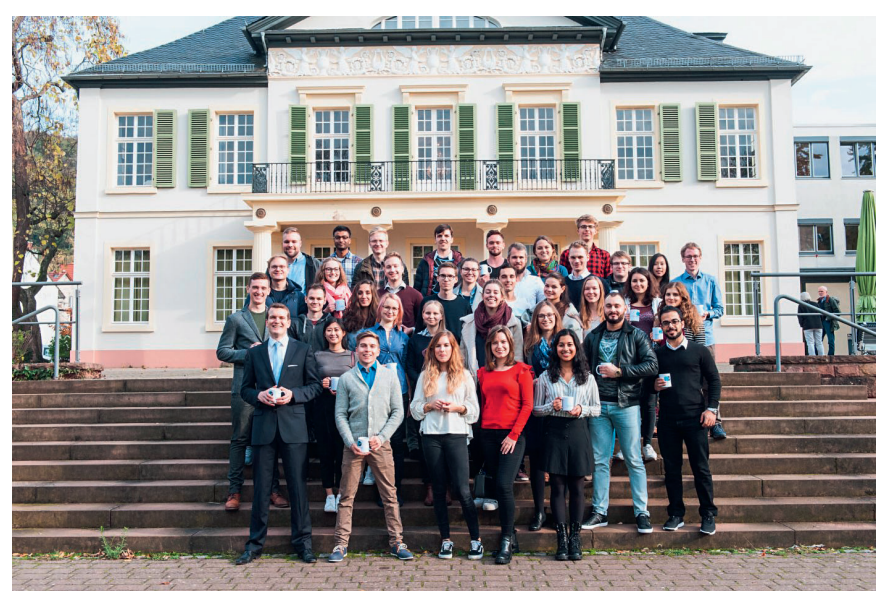

Gruppenleiter aus Deutschland 2017. @ Aufklärung gegen Tabak

fälle, Herzinfarkte oder generelle Herzschwäche - das sind auch alles weit verbreitete Folgen des Rauchens. Da fällt vielleicht doch jedem jemand aus dem eigenen Umfeld oder der Familie ein, der an so einer Krankheit leidet und der auch geraucht hat.

Der Verein «Aufklärung gegen Tabak» setzt sich unter anderem dafür ein, dass angehende Ärzte schon im Studium lernen, rauchende Patienten professionell zu beraten und ihnen bei der Rauchentwöhnung zu helfen. Wie gehen Sie da vor? Wir haben festgestellt, dass in der curricularen Lehre an den meisten Medizinfakultäten die Rauchentwöhnung überhaupt keine Rolle spielt. Meistens hören wir nur, welche Folgeerkrankungen das Rauchen mit sich bringt - aber wir kommen nicht darauf zu sprechen, wie wir das verhindern können. Dieser Präventionsaspekt sollte in unserem Studium eigentlich eine viel größere Rolle spielen. Eine Befragung hat vor einigen Jahren ergeben, dass sehr viele Studenten im letzten Jahr sich nicht kompetent fühlen, Patienten mit Nikotinabhängigkeit adäquat zu behandeln. Daraus ist unser Wahlfach entstanden, das wir inzwischen in Kooperation mit verschie- 
denen Kliniken an neun Fakultäten durchführen. Damit wollen wir Medizinstudenten darauf vorbereiten, in den Kontakt mit Patienten zu treten, die rauchen und die vielleicht schon den ersten Herzinfarkt hinter sich haben. Die werden dann vor die Wahl gestellt, ob sie dauerhaft Tabletten nehmen oder ihren Lebensstil ändern möchten. Wir wollen den Studierenden bewusstmachen, dass der Rauchstopp auf jeden Fall eine wirksamere Therapie ist als jede Tablette. Generell sollte man jeden Patienten nach seinem Rauchverhalten fragen. Bei Nichtrauchern weiß man dann, dass man an dieser Schraube nicht mehr drehen muss.

Außerdem bieten Sie Präventionsprojekte an Schulen an. Wie machen Sie Schüler auf die negativen Folgen des Rauchens aufmerksam?

Es gibt ja schon viele Präventionsprojekte an Schulen. Einige davon sehen wir eher kritisch: So glauben wir, dass z.B. Gespräche mit Lungenkrebs-Patienten die Jugendlichen eher nicht vom Rauchen abhalten - sie distanzieren sich eher davon, wenn eine ältere, kranke Person in die Schule kommt. Sie sehen nicht, was das mit ihnen zu tun hat, wenn sie ab und zu zur Zigarette greifen. Wenn wir in Schulen gehen, sprechen wir kaum über Krebserkrankungen - das ist für die Schüler zu weit weg. Unser Fokus liegt eher auf den kurzfristigen Folgen des Rauchens. Dazu haben wir auch die kostenlose App «Smokerface» entwickelt, die die kosmetischen Folgen des Rauchens am eigenen Gesicht deutlich macht.

\section{Wie funktioniert diese App?}

In der App macht man ein Selfie und kann dann angeben, wie viele Jahre man sein Gesicht in die Zukunft altern lassen möchte. Dann kann man die Fotos als Raucher und als Nichtraucher vergleichen. Das soll den Jugendlichen zeigen, welche Effekte das Rauchen auf ihr eigenes Aussehen haben kann. Das sind zum Beispiel Pickel, frühzeitige Faltenbildung oder die Verfärbung der Zähne. Das ruft bei den Schülern relativ heftige Reaktionen hervor - es entsteht auch ein Gruppendruck, wenn man zum Beispiel in der Klasse die Fotos seiner engsten Freunde sieht. Gerade in diesem Alter sind die eigene Schönheit und das eigene Selbstwertgefühl viel relevanter als das Risiko, in 40 oder 50 Jahren an Lungenkrebs zu erkranken. Aktuell betreuen wir zwei wissenschaftliche Studien, bei denen wir evaluieren, welchen Erfolg unsere Präventionsmaßnahmen tatsächlich haben.

Ihre Initiative hat auch noch eine zweite App entwickelt: «Smokerstop». Wie funktioniert diese App?

«Smokerstop» richtet sich an Raucher aller Altersgruppen. Die App soll helfen, mit dem Rauchen aufzuhören und wird dafür z.B. für die Mitarbeiter von BMW eingesetzt. Der grundsätzliche Wille dazu muss natürlich da sein. Wenn man aufhören will, gibt es immer wieder die Momente, in denen man das Bedürfnis hat, zu rauchen oder in denen man früher zur Zigarette gegriffen hätte. Und da braucht man Unterstützer: Am besten ist es, wenn Freunde oder Kollegen einen in solchen Momenten motivieren, nicht zu rauchen. Wenn man da niemanden hat, hilft die App, indem sie einem motivierende Nachrichten anzeigt. Da gibt man an, wie viel und was man bisher geraucht hat. Und die App zeigt einem dann zum Bei-
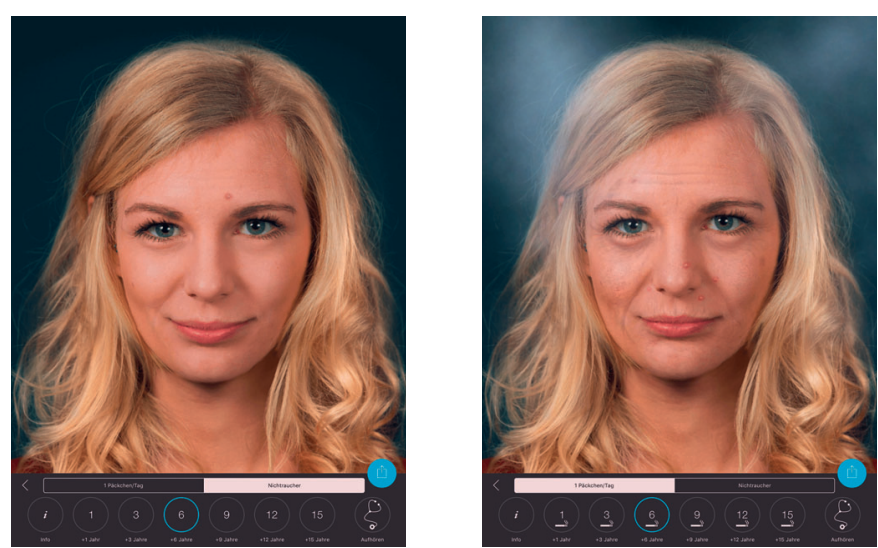

Wie sieht das eigene Gesicht in sechs Jahren aus - als Nichtraucher (links) oder als Raucher mit einem Päckchen Zigaretten täglich (rechts)? Die App «Smokerface» lässt Selfies optisch altern. (@ Aufklärung gegen Tabak e.V.

spiel an, wieviel Geld man schon gespart hat. Gleichzeitig bekommt man auch die positiven körperlichen Effekte angezeigt: Wie lange dauert es, bis sich mein Blutdruck normalisiert hat? Wann haben sich meine Lungen erholt? Wie lange dauert es, bis mein Risiko für Herz-Kreislauf-Erkrankungen stark gesunken ist? Wenn man das Verlangen nach einer Zigarette hat, sieht man so, warum es sich lohnt, diese Zigarette nicht zu rauchen.

\section{Wie hat sich der Verein seit der Gründung im Jahr 2012} entwickelt?

Der Gründer, Titus Brinker, hat damals als Medizinstudent in Gießen von Anfang an eine Expansion des Projekts geplant. Das Projekt konnte sich schnell auch an anderen Standorten etablieren. Mittlerweile sind wir an den meisten Medizinfakultäten in Deutschland vertreten. Wir expandieren jetzt sogar ins Ausland: «Aufklärung gegen Tabak» existiert inzwischen in zwölf Ländern - teils unter dem Namen «Education against tobacco». Ich denke, unser Projekt wird auch in Zukunft noch weiter expandieren - sowohl bundesweit als auch international.

Das Programm «Aufklärung gegen Tabak» wurde 2012 von Dr. Titus Brinker ins Leben gerufen, der damals im dritten Semester an der medizinischen Fakultät in Gießen studierte. Heute klären über 1500 Medizinstudierende von 28 deutschen, 4 österreichischen und 2 Schweizer Universitäten pro Jahr ehrenamtlich 23 800 Schüler über die Folgen des Rauchens auf - außerdem sind unter dem Namen «Education against tobacco» fast 60 Fakultäten im nicht-deutschsprachigen Ausland Teil der Initiative. Mehr Infos unter www.gegentabak.de.

\section{Originalquelle}

Stefanie Hanke für Operation Karriere - Das Karriereportal und der Kongress für angehende und junge Ärzte, www.operation-karriere.de (Direktlink für Onlineversion: https://www.operation-karriere.de/karriereweg/medizinstudium/medizinstudenten-engagieren-sich-aufklaerung-gegen-tabak-ev-html)

OPERATION 


\section{Medizinrecht \\ Übernahmeverschulden - was versteht man darunter?}

Der Arzt darf die Behandlung eines Patienten nur übernehmen, wenn er über die hierfür erforderliche persönlich fachliche Qualifikation und die nach dem Stand der medizinischen Wissenschaft erforderliche technisch-apparative Ausstattung verfügt.

Ist dies nicht gewährleistet, muss er die Behandlung ablehnen und den Patienten an einen ausreichend qualifizierten Kollegen oder ein apparativ ausreichend ausgestattetes Krankenhaus überweisen. Überschreitet der Arzt seine fachliche Kompetenz, ist er mit der geplanten Behandlung überfordert oder ist die zur Verfügung stehende technisch-apparative und personelle Ausstattung unzureichend, liegt schon in der Übernahme oder Fortsetzung der Behandlung ein Übernahmeverschulden (Sorgfaltspflichtverletzung) vor. Den Beweis, dass der eingetretene Schaden beim Patienten nicht auf die festgestellten Mängel in der persönlichen Qualifikation oder der Ausstattung zurückzuführen ist, muss dann im Prozess der Arzt führen.

In der Praxis fallen hierunter die sogenannten Anfängeroperationen oder die Behandlung durch übermüdete Ärzte. Insbesondere der in der Weiterbildung befindliche Arzt muss sich selbstkritisch ein Bild über die eigenen Fähigkeiten machen. Er ist verpflichtet, wenn er den notwendigen Weiterbildungsstand und die Erfahrung noch nicht erreicht hat, einen erfahrenen Kollegen hinzuzuziehen oder aber die Vornahme des Eingriffs abzulehnen, da er sonst für die durch die fehlerhafte Behandlung entstandenen Schäden haftet. Die Behandlung dürfte nur dann von einem Anfänger durchgeführt werden, wenn dies unter Anleitung eines Facharztes erfolgt, der sofort eingreifen kann.

Als Folge der möglichen Haftung für ein Übernahmeverschulden ergibt sich auch die Verpflichtung des Arztes (z.B. des Hausarztes) an einen Facharzt/Spezialisten zu überweisen oder den Rat eines Konsiliarius einzuholen, wenn die gebotene Behandlung die Fähigkeiten und Kenntnisse des behandelnden Arztes übersteigt.

\section{ZUM AUTOR}

Maximilian Broglie, Seniorpartner in der Kanzlei Broglie, Schade \& Partner GbR, Wiesbaden, ist Fachanwalt für Sozial- und Medizinrecht. Neben seiner Tätigkeit als Geschäftsführer der Deutschen Gesellschaft für Innere Medizin e.V. (DGIM), wirkt er zudem bei zahlreichen Buch- und Zeitschriftenprojekten mit. 